\title{
Fabrication of Graphene Nanomesh FET Terahertz Detector
}

\author{
Yuan Zhai ${ }^{1}$, Yi Xiang ${ }^{1}$, Weiqing Yuan ${ }^{2}$, Gang Chen ${ }^{2}$, Jinliang Shi ${ }^{1}$, Gaofeng Liang ${ }^{2}$, Zhongquan Wen ${ }^{2}$ \\ and Ying $W \mathbf{u}^{1, *}$ \\ 1 Intelligent Technology and Engineering, Chongqing University of Science and Technology, \\ Chongqing 401331, China; cquzhy@cqust.edu.cn (Y.Z.); xiangyicq@163.com (Y.X.); 13608335626@163.com (J.S.) \\ 2 College of Optoelectronic Engineering, Chongqing University, Chongqing 400044, China; \\ ywqwiki@gmail.com (W.Y.); gchen1@cqu.edu.cn (G.C.); lgf@cqu.edu.cn (G.L.); wenzq@cqu.edu.cn (Z.W.) \\ * Correspondence: wuying1992@cqust.edu.cn
}

check for

updates

Citation: Zhai, Y.; Xiang, Y.; Yuan, W.; Chen, G.; Shi, J.; Liang, G.; Wen, Z.; Wu, Y. Fabrication of Graphene Nanomesh FET Terahertz Detector. Micromachines 2021, 12, 641. https:// doi.org/10.3390/mi12060641

Academic Editor: Francesco La Via

Received: 25 February 2021

Accepted: 17 May 2021

Published: 31 May 2021

Publisher's Note: MDPI stays neutral with regard to jurisdictional claims in published maps and institutional affiliations.

\begin{abstract}
High sensitivity detection of terahertz waves can be achieved with a graphene nanomesh as grating to improve the coupling efficiency of the incident terahertz waves and using a graphene nanostructure energy gap to enhance the excitation of plasmon. Herein, the fabrication process of the FET THz detector based on the rectangular GNM (r-GNM) is designed, and the THz detector is developed, including the CVD growth and the wet-process transfer of high quality monolayer graphene films, preparation of r-GNM by electron-beam lithography and oxygen plasma etching, and the fabrication of the gate electrodes on the $\mathrm{Si}_{3} \mathrm{~N}_{4}$ dielectric layer. The problem that the conductive metal is easy to peel off during the fabrication process of the GNM THz device is mainly discussed. The photoelectric performance of the detector was tested at room temperature. The experimental results show that the sensitivity of the detector is $2.5 \mathrm{~A} / \mathrm{W}$ (@3 THz) at room temperature.
\end{abstract}

Keywords: graphene nanomesh; $\mathrm{Si}_{3} \mathrm{~N}_{4}$ dielectric layer; FET; terahertz detector

\section{Introduction}

Graphene, a conjugated carbon sheet arranged in a 2D hexagonal lattice [1] and an important alternative to extend the validity of Moore's law of electrons in semiconductors [2], has good transmission performance, a larger volume miniaturization space and a lower cost by virtue of ultrahigh electron mobility and ultrathin material thickness [3-5]. Due to the ultrathin planar structure of graphene, the performances of graphene-based field-effect transistor (GFET) devices are not obviously reduced when they shrink in size. The fabrication of the device is compatible with current CMOS technology, making GFET a highly competitive choice for high-performance, high-integration chips in the future [6-8].

At present, terahertz technology is widely used in many areas, such as defense, medical diagnosis, security monitoring, communication technology and space exploration. The development of terahertz technology has put forward higher requirements for terahertz detectors. However, due to the inherent limitations of electron velocity, the performance of traditional microwave electronic transistors decreases rapidly as the frequency approaches to the terahertz $(\mathrm{THz})$ band $(>0.1 \mathrm{THz})$. It is also difficult for the infrared optical devices to have good applications at frequencies below $20 \mathrm{THz}$ [9]. The special position of $\mathrm{THz}$ in the electromagnetic spectrum (both electronic and optical devices are involved) poses a severe challenge to the modern solid-state devices [10]. With high carrier mobility, tunable electronic properties and unique photoelectric properties, graphene provides a new idea for the research of terahertz direct detectors. Terahertz detectors based on the GFET structure are developed and reported [6,7,11-13].

Although some methods can open the band gap of graphene, such as using graphene nanoribbons and double-layer graphene or applying stress to graphene, due to a series of challenges it has not been able to achieve mass production in the existing semiconductor process [11-14]. So far, no mature solid-state devices have been widely used in the terahertz 
band. This study aims to solve the problem encountered in the process of graphene transfer and lamination. In the paper, high quality graphene materials were prepared by chemical vapor deposition (CVD). The large-area and uniform graphene nanomesh structure was fabricated by electron-beam lithography (EBL). The fabrication process of the terahertz detector based on the graphene nanomesh was designed and the terahertz detector was developed.

\section{Fabrication Process of Graphene Terahertz Detector}

Based on the principles of the graphene FET terahertz detector, the pitch size and dimensions of the graphene nanomesh and structural parameters are decided and described in Table 1.

Table 1. Parameters of the graphene FET terahertz detector.

\begin{tabular}{ll}
\hline Parameters of Graphene & Value \\
\hline Minimum width of nanostructures & $30 \mathrm{~nm} \sim 60 \mathrm{~nm}$ \\
Period of structural array & $1 \mu \mathrm{m}$ \\
Channel length & $14 \mu \mathrm{m}$ \\
Channel width & $60 \mu \mathrm{m}$ \\
Thickness of dielectric layer & $60 \mathrm{~nm}$ \\
\hline
\end{tabular}

According to the device structure we designed, and the micro-nano processing platform of the National Center for Nanoscience and Technology of China, the overall fabrication process of the device is divided into the following five steps as shown in Figure 1.

(a)

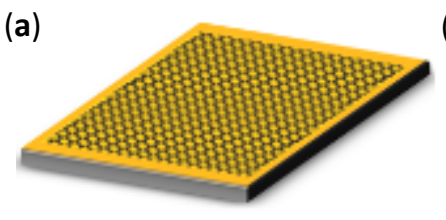

(b)

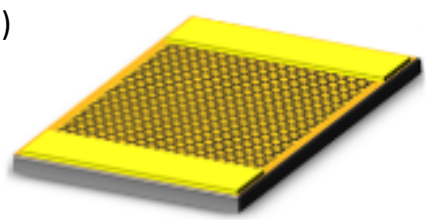

(d)

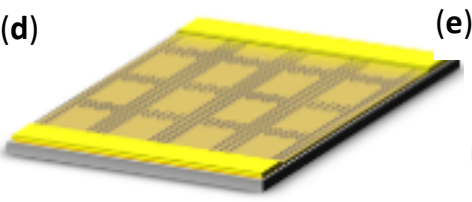

(e)

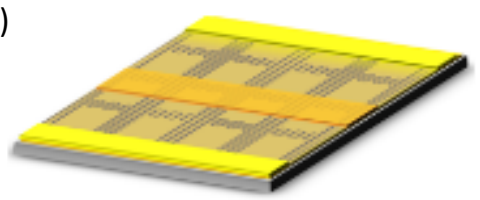

(c)

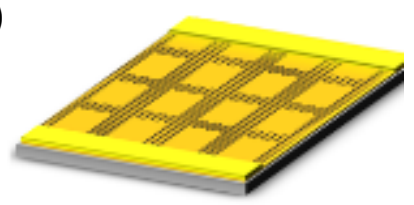

Figure 1. The fabrication process of the graphene terahertz field-effect transistor (FET) detector. (a) Chemical vapor deposition (CVD) preparation and substrate transfer of graphene. (b) Fabrication of source and drain electrodes. (c) Fabrication of graphene nanogrid by electron-beam lithography (EBL). (d) CVD deposition of the $\mathrm{Si}_{3} \mathrm{~N}_{4}$ dielectric layer. (e) Fabrication of the gate electrode.

\subsection{Chemical Vapor Deposition (CVD) and Transfer of Graphene}

Graphene film is prepared on copper foil $(2 \mathrm{~cm} \times 2 \mathrm{~cm} \times 25 \mu \mathrm{m}$ in volume) by CVD $[4,15,16]$, and then transferred to the prepared $\mathrm{Si} / \mathrm{SiO}_{2}$ substrate. Firstly, the desired copper foil substrate was obtained with the method of chemical immersion cleaning combined with electrochemical polishing. Then, graphene crystals were grown in a singletemperature-zone CVD tube furnace. The temperature was raised in a low-pressure environment (the pressure was set at 600-800 mTorr), and $\mathrm{H}_{2}$ was introduced when the temperature was raised. Under the condition of $1050{ }^{\circ} \mathrm{C}$, the copper foil substrate was annealed for $1.5 \mathrm{~h}$ and maintained at this temperature, and $\mathrm{H}_{2}$ (catalyst) and $\mathrm{CH}_{4}$ (carbon source) were introduced for chemical reaction preparation. The reaction time was controlled within half an hour. After the graphene film was formed, the PMMA was coated as the protective layer. The thickness of the PMMA is about $200 \mathrm{~nm}$ when the spin speed is 4000 RPM and the concentration of the PMMA is 6\%. Ferric chloride and 
hydrochloric acid were used as etching solutions to soak the copper foil substrate and some inorganic impurities. After copper foil etching, the PMMA/graphene was cleaned with deionized water. The selected substrate is a highly doped p-type silicon with a thickness of $525 \mu \mathrm{m}$. The thickness of the oxide layer above the silicon wafer is $285 \mathrm{~nm}$. Before the transfer, the substrate was cleaned with deionized water and then dried with nitrogen. The PMMA/graphene was directly transferred to the substrate. At last, the PMMA was dissolved and removed in an acetone solution. In order to prevent graphene damage and avoid using ultrasound, the PMMA was removed by $80^{\circ} \mathrm{C}$ water bath heating.

\subsection{Fabrication of the Source and Drain Electrodes}

Graphene film is essentially a single layer of carbon atoms. During the process design of the device, graphene damage should be avoided as far as possible. Therefore, the stripping process is chosen for electrode evaporation in order to avoid damage to the graphene caused by strong acid etching solutions in the electrode manufacturing process.

However, the metal pattern formed on graphene by the stripping process is easy to fall off. The main reason is that the bonding between graphene and substrate is very low due to the van der Waals force. When the metal film is evaporated on graphene, the metal does not directly make contact with the substrate, and graphene may also act as a "stripping adhesive" in the stripping process to strip the metal directly. The phenomenon of metal peeling is observed obviously, especially in the location of the large-area metal pad as shown in Figure 2.

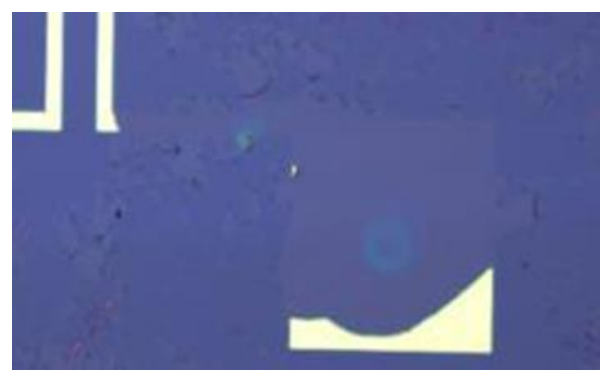

Figure 2. Metal shedding occurs when the graphene acts as a "stripping adhesive".

In order to solve this problem, a process of etching graphene is added on the basis of the conventional stripping process, and then the metal evaporation stripping process is carried out twice. The optimized process is shown in Figure 3.

Before spin-coating, the graphene substrate is pretreated and soaked in acetone at room temperature for half an hour. After that, the graphene substrate is cleaned with isopropanol and deionized water, and dried with nitrogen successively. Next, the prebaking temperature for the substrate is set to $120^{\circ} \mathrm{C}$ and the heating time is $3 \mathrm{~min}$. Then, a $4 \%$ concentration of PMMA (950 k) is selected as the positive glue to spin onto the substrate with a spin-coating speed of 5000 RPM. After spin-coating, the substrate is post-baked at $150{ }^{\circ} \mathrm{C}$ for $2 \mathrm{~min}$, as shown in Figure $3 \mathrm{a}$.

Figure $3 \mathrm{~b}$ shows that the positive photoresist stripping process is used to expose the pattern of the metal wire and pad, in which the graphene would be removed, excluding the part of the source and drain electrodes located in the central part framed in the red dotted line. Figure 4 shows the layout pattern for electron-beam direct writing with an exposure dose of $850 \mu \mathrm{C} / \mathrm{cm}^{2}$. In order to improve the exposure efficiency, the electron-beam step is set at $50 \mathrm{~nm}$. 


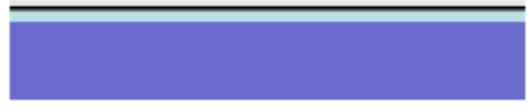

(a)

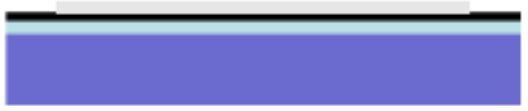

(c)

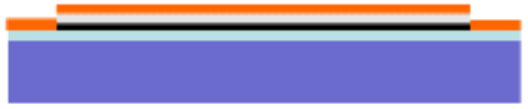

(e)

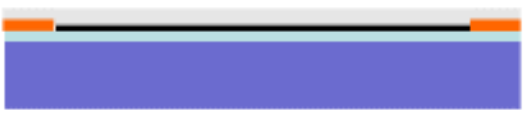

(g)

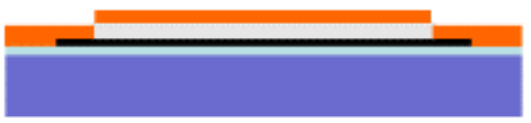

(i)

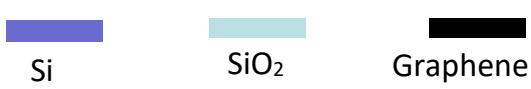

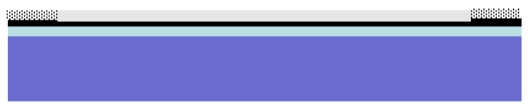

(b)

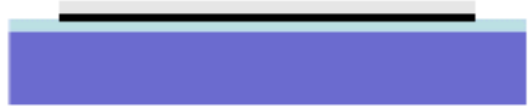

(d)

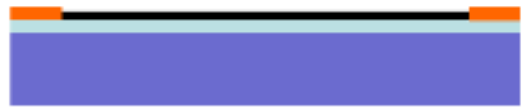

(f)

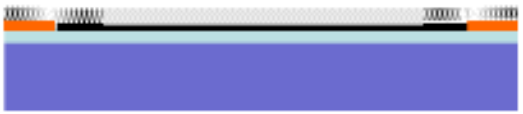

(h)

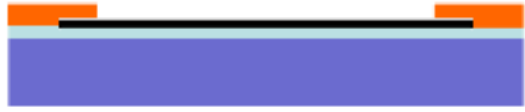

(j)

PMMA Positive lithography Metal

Figure 3. A schematic diagram of the source and drain electrode production process. (a) Spincoating positive photoresist (b) Electron-beam lithography (c) Positive photoresist development (d) ICP etching (e) First evaporation of metal (f) Stripping (g) Spin-coating positive photoresist (h) Electron-beam lithography (i) Second evaporation of metal (j) Stripping.

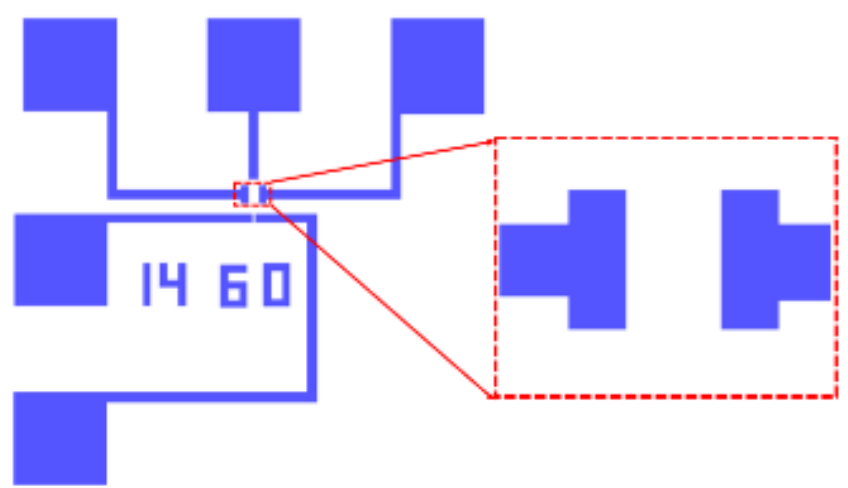

Figure 4. The layout of etching graphene under metal.

Figure $3 c$ shows the result of the positive photoresist development. The developer was a mixture of MIBK: IPA = 1:3. After $100 \mathrm{~s}$ of developing time, the substrate was then soaked in isopropanol for $50 \mathrm{~s}$. In order to avoid the stripping effect of graphene upon the metal, oxygen plasma was used to etch the graphene with an etching time of $8 \mathrm{~s}$ and an etching power of $150 \mathrm{~W}$ under a pressure of $5 \mathrm{~Pa}$. The development result is shown in Figure 3d. Then, the first evaporated metal of $8 \mathrm{~nm} / 40 \mathrm{~nm}$ Ti/Au, shown in Figure 3e, could make contact with the $\mathrm{SiO}_{2} / \mathrm{Si}$ substrate to enhance the adhesion force. Meanwhile, the useless graphene easily fell off. Therefore, water bath heating and longer soaking time can be used to enhance the stripping effect without using ultrasound. The stripping result is shown in Figure $3 \mathrm{f}$.

The same PMMA positive photoresist was used for spin-coating again. The peeling effect of the subsequent process could be enhanced by appropriately increasing the thickness of the spin-coating, as shown in Figure 3g. The source and drain electrodes are designed 
and shown in the enlarged picture in Figure 5. This scheme causes the metal to directly make contact with the substrate to enhance the adhesion of the metal, and it can also reduce the contact resistance by making the source and drain electrodes directly make contact with graphene, as shown in Figure $3 \mathrm{~h}$. The parameters of electron-beam exposure are the same as those of the first exposure.

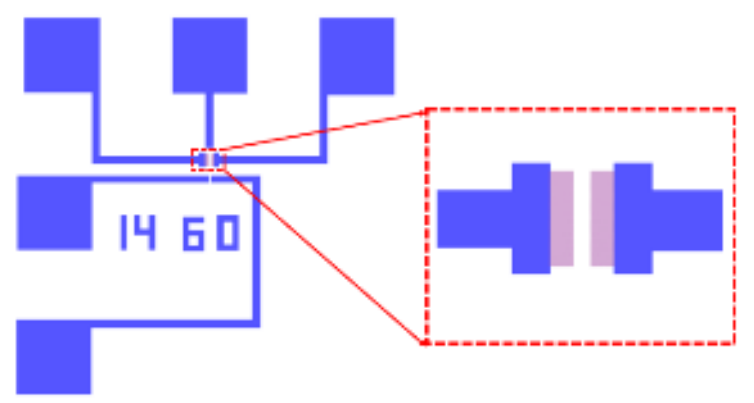

Figure 5. The layout of evaporating metal for the source and drain.

The evaporated metal is Au with a thickness of $100 \mathrm{~nm}$ as shown in Figure 3i, including the patterns of wire and pad, and source and drain electrodes. In order to ensure the continuity of the whole metal pattern, the evaporation thickness is required to exceed the first overall thickness. Figure $3 \mathbf{j}$ shows the source and drain electrodes and metal pads obtained by the same stripping method presented above.

\subsection{Fabrication of Graphene Nanomesh by EBL and OPE}

In order to obtain the large-area and uniform graphene nanogrid, the micro-nano fabrication method of electron-beam lithography (EBL) and oxygen plasma etching (OPE) was used to obtain the corresponding size of graphene nanogrid structure materials. Firstly, a large-area single-layer graphene was grown by chemical vapor deposition on a copper substrate. It was then transferred onto heavily doped p-type Si substrates with a $285 \mathrm{~nm}$ $\mathrm{SiO} 2$ layer using polymethyl methacrylate (PMMA)-assisted wet-transfer techniques. The silicon wafer (substrate size $1.2 \mathrm{~cm} \times 1.2 \mathrm{~cm}$ ) with the transferred graphene film was soaked in an acetone solution for $12 \mathrm{~h}$, then soaked in isopropanol, slightly washed with deionized water, and finally dried $\left(<80^{\circ} \mathrm{C}\right)$. Then, the PMMA with a concentration of $4 \%$ was used as a resist, and the spin-coating speed was set at 5000-6000 RPM (the corresponding coating thickness was about $200 \mathrm{~nm}$ ). For different structures and sizes of GNM, the corresponding exposure dose was determined and selected for electron-beam exposure. At last, a graphene nanomesh structure was fabricated by oxygen plasma etching. The layout of the graphene channel between the source and drain electrodes is shown in Figure 6a, which was processed by EBL [17]. The exploration of the graphene nanomesh is shown in the right image of Figure 6b. After the electron-beam direct writing exposure process is completed, the channel image of the device is obtained and shown on the left of Figure $6 \mathrm{~b}$.

(a)

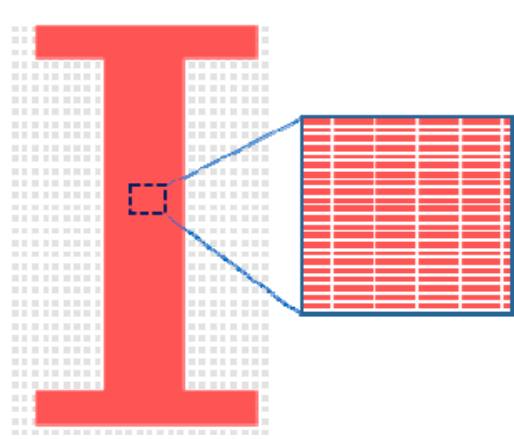

(b)

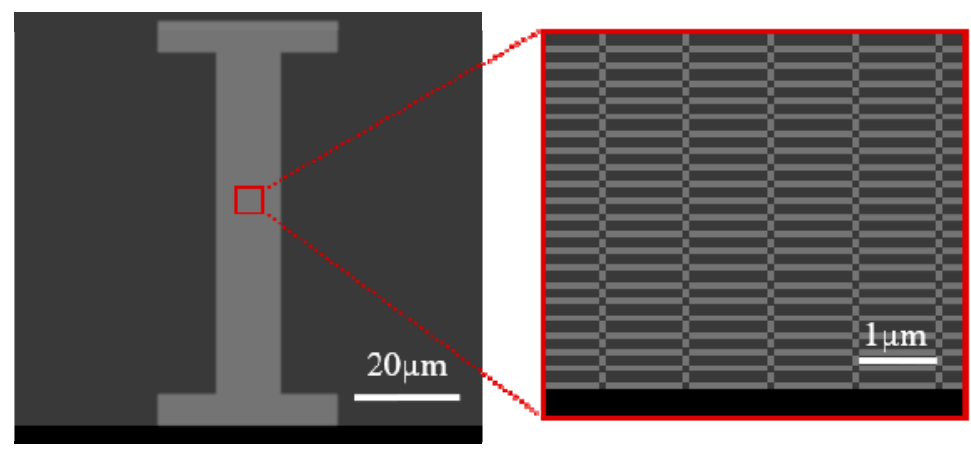

Figure 6. (a) Layout of rectangular graphene nanomesh (r-GNM) channel (b) SEM of rectangular graphene nanomesh (r-GNM) channel. 
SEM images of the graphene nanomesh structure after oxygen plasma etching are shown in Figure 7. It can be seen that the fabricated graphene nanogrid can maintain the integrity of the periodic structure in the large-area.

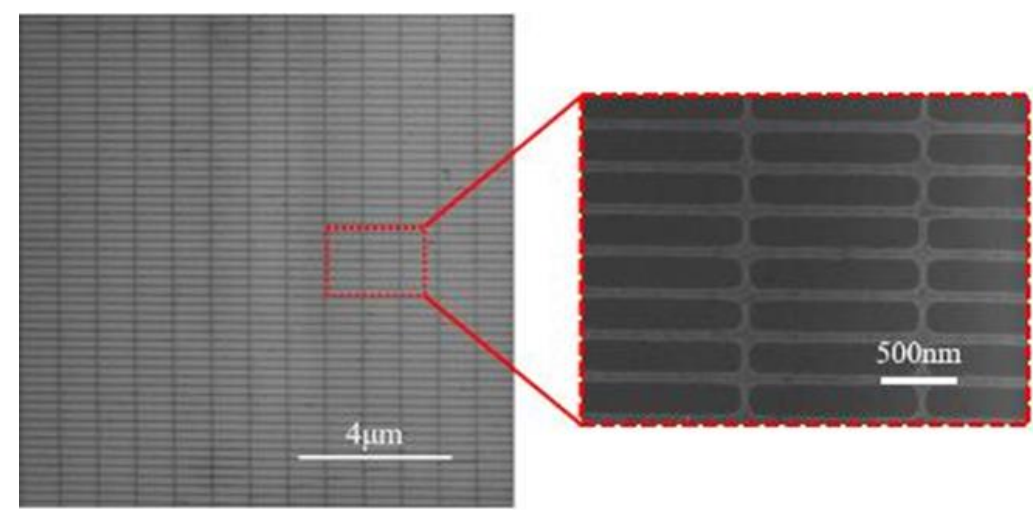

Figure 7. SEM images of the graphene nanomesh structure after oxygen plasma etching.

\subsection{CVD Deposition of the Dielectric Layer}

As the device will work in THz frequency bands, the dielectric constant of the gate dielectric layer under the gate electrode should be as large as possible. For graphene that has been processed into nanostructure, the bonding ability with the dielectric layer on the surface of graphene and the question of easy damage should be considered carefully in the subsequent processes.

Therefore, plasma-enhanced chemical vapor deposition (PECVD) was chosen to deposit $\mathrm{Si}_{3} \mathrm{~N}_{4}$ on the surface of the graphene nanomesh as the dielectric layer. Because the combination of $\mathrm{Si}_{3} \mathrm{~N}_{4}$ and graphene is much better than that of a silicon oxide dielectric layer created by the thermal oxidation process, the dielectric constant of $\mathrm{Si}_{3} \mathrm{~N}_{4}(\varepsilon=6.6)$ is much higher than that of silicon oxide $(\varepsilon=3.9)$, and $\mathrm{Si}_{3} \mathrm{~N}_{4}$ has a higher polarized photophonon frequency, which can reduce the phonon scattering of the graphene conductive channel and is conducive to the photoelectric application of graphene [18]. Simultaneously, the PECVD process worked at a low-temperature environment, which could effectively prevent the damage of graphene. The gas source of $\mathrm{Si}_{3} \mathrm{~N}_{4} \mathrm{CVD}$ was the inert gas of $\mathrm{N}_{2}$ and $\mathrm{NH}_{3}$. In addition, the power source was a low-density plasma with a power of only $40 \mathrm{~W}$. These factors could ensure that the damage to graphene is minimized in the manufacturing process of the dielectric layer.

The growth process of the $\mathrm{Si}_{3} \mathrm{~N}_{4}$ dielectric layer is shown in Figure 8. The result of spin-coating positive photoresist is shown in Figure 8a. The rotation speed was set at 4000-5000 RPM to spin-coat with the PMMA $950 \mathrm{k}$ positive photoresist and control the photoresist thickness above $200 \mathrm{~nm}$. The pre-baking and post-baking time are 1-2 and $2 \mathrm{~min}$, respectively. The pre-baking and post-baking temperatures are 120 and $150^{\circ} \mathrm{C}$, respectively. As the pattern of the dielectric layer is simple and the requirement of dimensional accuracy is low, only accurate alignment is required for electron-beam direct writing. The exposure dose of electron beam was $850 \mu \mathrm{C} / \mathrm{cm}^{2}$, and the step setting was $50 \mathrm{~nm}$. The fabrication of EBL is shown in Figure 8b. The mixture of MIBK and IPA (1:3) was used as developer. The positive photoresist development time was $100 \mathrm{~s}$, and isopropanol was used for fixing for 30 s. After the development was obtained, as shown in Figure $8 \mathrm{c}$, the $\mathrm{Si}_{3} \mathrm{~N}_{4}$ was grown by using the $\mathrm{Si} 500 \mathrm{D}$ PECVD equipment (SENTECH). $\mathrm{SiH}_{4} / \mathrm{N}_{2}$ and $\mathrm{NH}_{3}$ were used as the gas source, and the reaction equation is:

$$
3 \mathrm{SiH}_{4}+4 \mathrm{NH}_{3} \rightarrow \mathrm{Si}_{3} \mathrm{~N}_{4}+12 \mathrm{H}_{2} \uparrow
$$




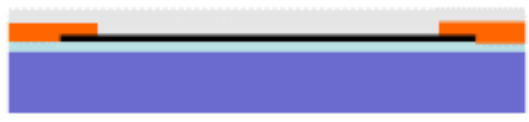

(a)

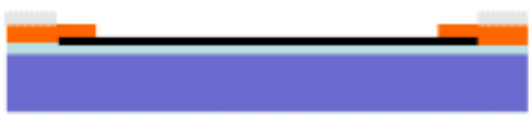

(c)

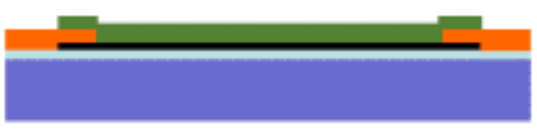

(e)

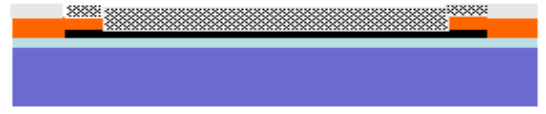

(b)

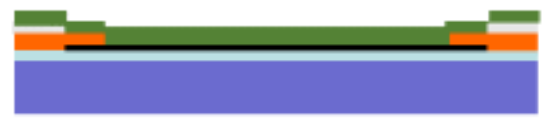

(d)

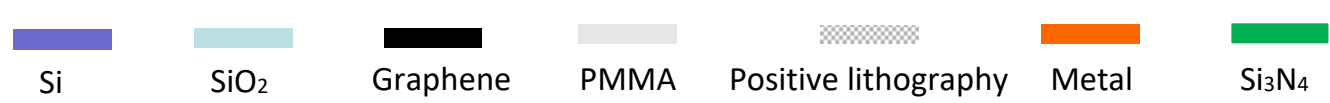

Figure 8. The preparation process of the dielectric layer. (a) Spin-coating positive photoresist (b) Electron-beam lithography (c) Positive photoresist development (d) CVD Si3N4 (e) Stripping.

While the RF power, temperature, pressure and deposition time are set to $40 \mathrm{~W}, 55^{\circ} \mathrm{C}$, $50 \mathrm{~Pa}$ and $1 \mathrm{~h}$, respectively, the thickness of the $\mathrm{Si}_{3} \mathrm{~N}_{4}$ film would be about $60 \mathrm{~nm}$, as shown in Figure 8d. The last step of the dielectric layer deposition is stripping, which used an acetone solution to remove the PMMA, and the result is shown in Figure 8e.

\subsection{Fabrication of the Gate Electrode}

After the fabrication of the dielectric layer $\mathrm{Si}_{3} \mathrm{~N}_{4}$, the graphene nanomesh was covered by silicon nitride, and the possibility of damage was reduced, as shown in Figure 9. Therefore, the conventional metal evaporation stripping process can be used for the fabrication of different gate electrodes. The specific process flow is shown in Figure 10.

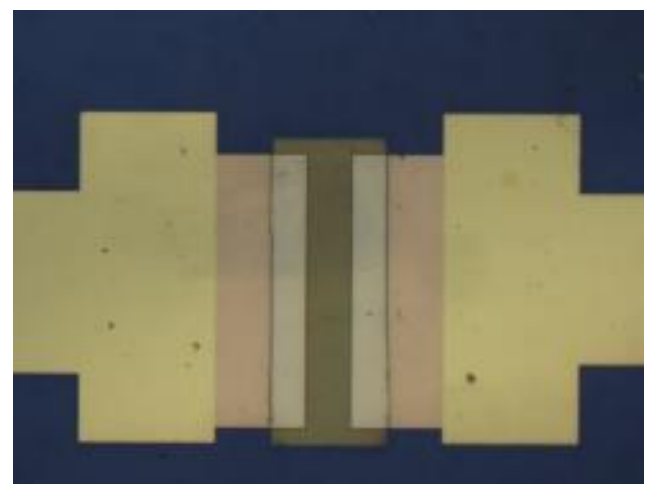

Figure 9. An optical photograph of the device after the fabrication of the dielectric layer.

Figure 10a shows the spin-coating result, among which PMMA (950 k) stock solution was used for spin-coating, the spin-coating speed was 4500 RPM, and the thickness of the adhesive was controlled above $200 \mathrm{~nm}$. The pre-baking and post-baking time both were $100 \mathrm{~s}$, and the temperatures were set to 80 and $150^{\circ} \mathrm{C}$, respectively. While the electron-beam exposure dose was set at $900 \mu \mathrm{C} / \mathrm{cm}^{2}$ and the step was set at $50 \mathrm{~nm}$, the overlay precision could be ensured for the electron-beam direct writing exposure of the gate electrode pattern. The EBL result is shown in Figure 10b. The positive development still used the mixture of MIBK and IPA (1:3) as developer in Figure 10c. The development time was $100 \mathrm{~s}$ and then isopropanol was used for fixing. The thickness of the evaporated gate metal should be as thin as possible, as shown in Figure 10d. Therefore, we evaporated 10/50 nm thick Ti/ Au metal, which is conducive to complete stripping. The PMMA adhesive was removed by immersing the substrate in an acetone solution to complete the stripping process, as shown 
in Figure 10e. A water bath with long-time soaking was used to ensure complete stripping, because due to the narrow metal spacing it was easy to cause incomplete stripping.

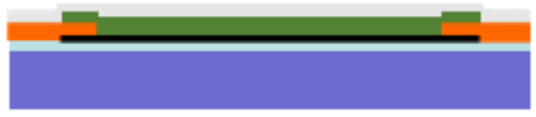

(a)

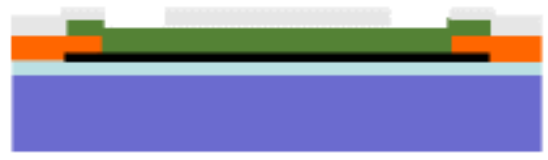

(c)

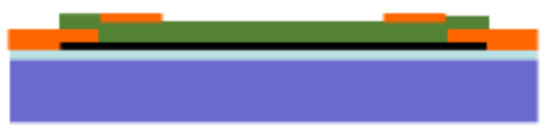

(e)

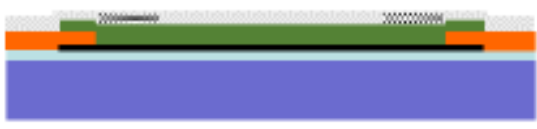

(b)

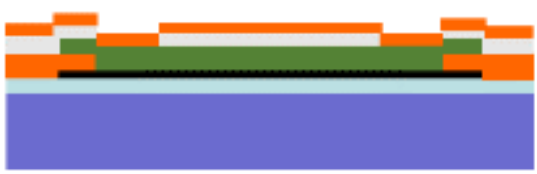

(d)

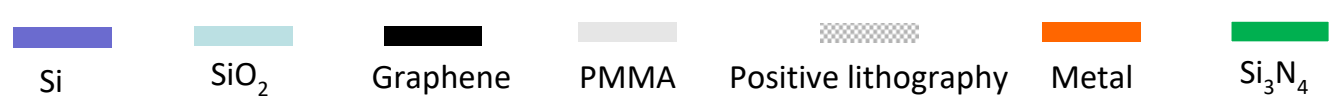

Figure 10. The process flow of the gate electrode. (a) Spin-coating positive photoresist (b) Electron-beam lithography (c) Positive photoresist development (d) Evaporating metal (e) Stripping.

Using the above process, we fabricated an r-GNM FET THz device with a graphene nanomesh size of $14 \mathrm{~nm} \times 60 \mathrm{~nm}$, which is shown in Figure 11 .
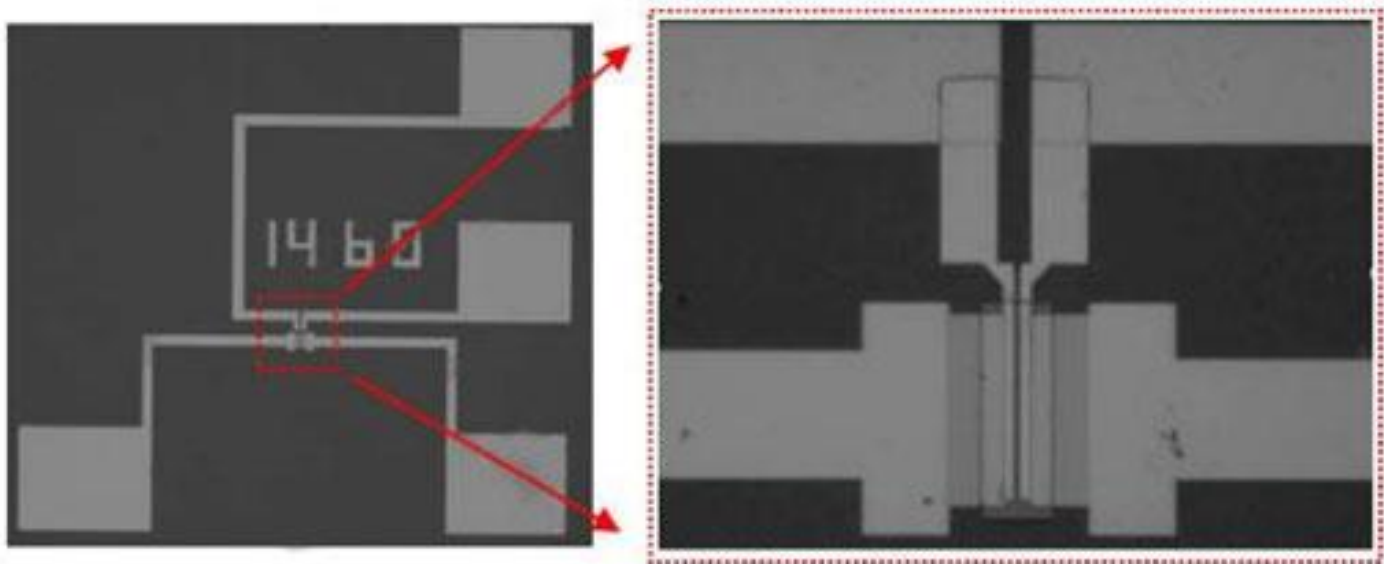

Figure 11. Optical photographs of the top gate FET device.

\section{Measurement Experiments}

According to the size of the silicon substrate, the PCB board is designed for the convenience of testing the electrical properties and terahertz detection of the FET device. Figure 12 shows that the metal pads of the device are connected to the pins of the PCB board by gold wires. 


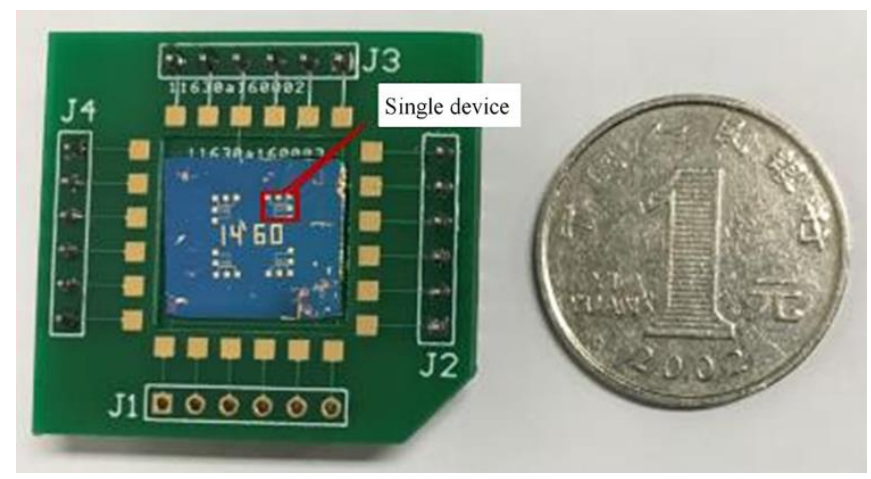

Figure 12. Physical drawings of devices on PCB.

\subsection{Electrical Transfer Characteristics}

The transfer characteristics of the Gr layer are shown in Figure 13. Figure 13a shows the transfer characteristics at $\mathrm{Vds}=2 \mathrm{~V}$ for the devices based on $\mathrm{r}$-GNMs with different neck widths of 30,40,50, and $60 \mathrm{~nm}$, from which we could determine the corresponding Ion/Ioff ratios of $\sim 40, \sim 25, \sim 5$, and $\sim 4$, respectively. The transfer characteristics for the devices based on c-GNMs with different neck widths of 30,40,50, and $60 \mathrm{~nm}$ are presented in Figure 13b. Comparing Figure 13a with Figure 13b, we can see that the conduction current of c-GNMs is much larger than that of r-GNMs (about two times). As a result of GNM being able to be viewed as an interconnected network structure of graphene, the actual area of c-GNM delivering current is greater than that of r-GNM, which leads to the current of c-GNM being greater than r-GNM under the same conditions. Additionally, the Ion/Ioff ratios of r-GNMs with different neck widths of 30, 40, 50, and $60 \mathrm{~nm}$ obtained were $\sim 100, \sim 25, \sim 8$, and $\sim 3$, respectively, indicating that the Ion/Ioff ratio of the GNM-based devices can be readily tuned by varying the neck width, which plays an important role in charge of transport properties.

(a)

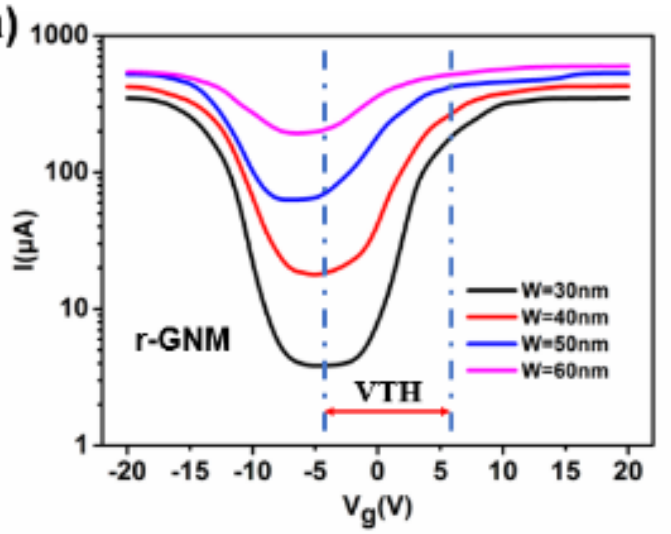

(b)

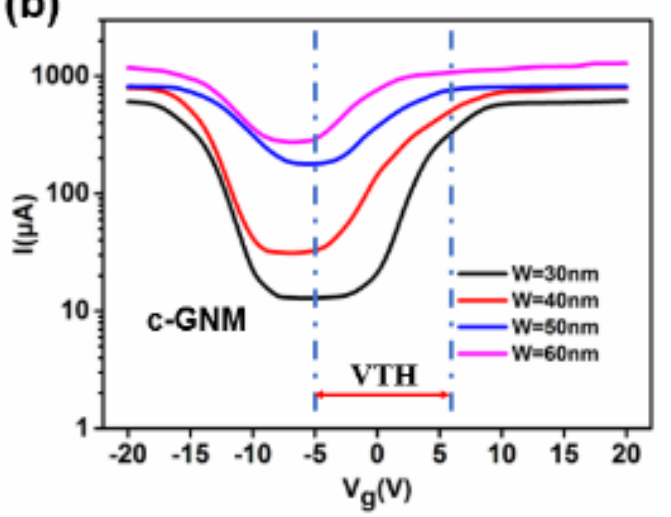

Figure 13. GNM transfer characteristic curves with dimensions of 30, 40, 50 and $60 \mathrm{~nm}$, respectively. (a) r-GNM (b) c-GNM.

\subsection{Terahertz Photocurrent Measurement}

The IR-30 steady-state infrared light source (HawkEye Technologies) was used as the light source for the $\mathrm{THz}$ response test. The excitation source of the light source was powered by $2.5 \mathrm{~V} \mathrm{DC}$ and could be regarded as a blackbody light source at a stable working state. A $3 \mathrm{THz}$ band-pass filter is used behind the light source to obtain a $3 \mathrm{THz}$ electromagnetic radiation wave. The BPF3.0 filter (TYDEX) was used to obtain the transmission spectrum, which is shown in Figure 14a. In order to obtain the influence of the gate voltage on the terahertz photocurrent, the gate voltage was changed to test the photocurrent for r-GNM with a $60 \mathrm{~nm}$ width. The gate voltage had a decisive effect on the dispersion of the graphene 
terahertz plasmon by changing the Fermi level, but the effect on the terahertz absorption of the graphene nanomesh was not obvious.

(a)

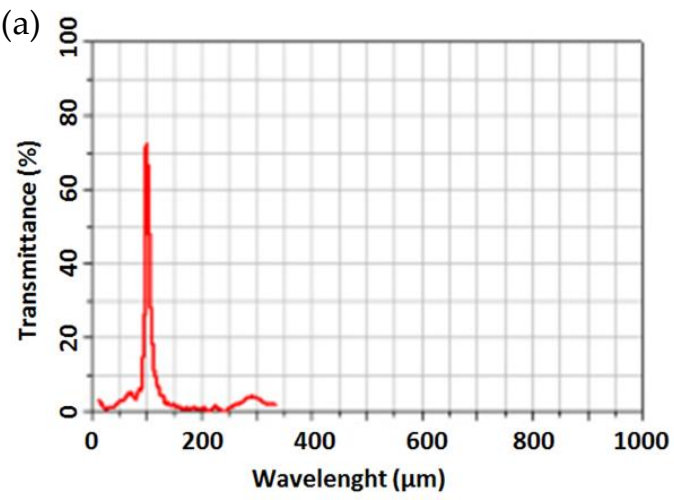

(b)

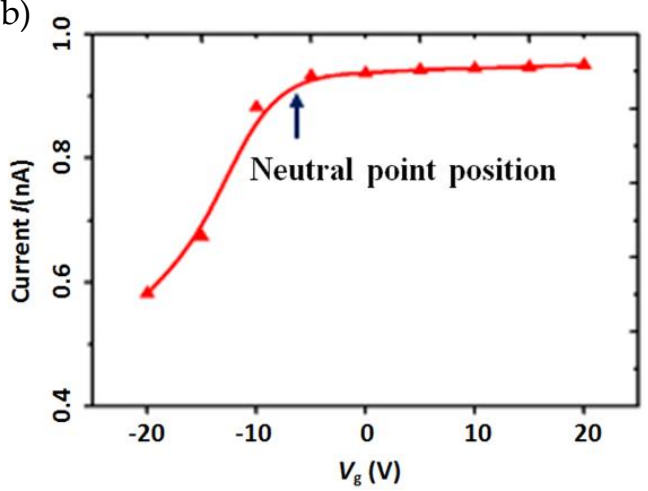

Figure 14. (a)The terahertz transmission of BPF3.0 (b) the curves of the photocurrent of the FET terahertz detector versus the gate voltage.

Figure $14 \mathrm{~b}$ shows that the increase in gate voltage increased the photocurrent, but the increase after the neutral point tends to be saturated. The reason is that the increase in gate voltage will also increase the Fermi energy level, and then affect the carrier concentration, so that the excitation of graphene plasmon is weak when the Fermi energy level is relatively low, resulting in a slightly small photocurrent. When the Fermi level increases to a certain extent, the saturation of the terahertz absorption makes the photocurrent no longer increase.

In order to further determine the response rate of the graphene nanomesh FET terahertz detector, the standard Golay cell GC-1P terahertz detector (TYDEX) was used to calibrate the power of the terahertz radiation source by replacing the graphene nanomesh FET terahertz detector in the same position. According to the voltage sensitivity formula:

$$
R_{V}=\frac{V}{P_{o}}
$$

where $R_{V}$ is the hardness of high Leghorn. When the reference frequency was $10 \mathrm{~Hz}$, the photovoltage signal intensity measured by the Golay GC-1P was $6.47 \mu \mathrm{W}$. Under this condition, the sensitivity of the Golay was $81.3 \mathrm{KV} / \mathrm{W}$, and the output optical power $P_{o}$ was about $0.08 \mu \mathrm{W}$. The current sensitivity formula of the detector is as follows:

$$
R_{i}=\frac{i}{P_{\text {in }}}
$$

where $R_{i}$ is the current sensitivity of the detector, $i$ is the detection current, and $P_{\text {in }}$ is the actual incident light power. The current sensitivity of the graphene terahertz FET detector is $12 \mathrm{~mA} / \mathrm{W}$ under $3 \mathrm{THz}$ radiation.

\section{Conclusions}

Monolayer graphene was grown on copper foil by CVD, and the high-quality graphene film was obtained by wet transfer on the $\mathrm{SiO}_{2} / \mathrm{Si}$ substrate. Then, aiming at the problems of electrode stripping, the method of metal evaporation and photoresist stripping twice was used to realize the source and drain electrode lamination of graphene, and enhance the adhesion of the metal wire and electrodes with the substance. Next, the graphene nanomesh with a large-area and uniform size was fabricated by EBL and oxygen plasma etching. Lastly, the conventional metal evaporation stripping process was used to fabricate the gate electrode on the $\mathrm{Si}_{3} \mathrm{~N}_{4}$ dielectric layer. In sum, the fabrication process of the terahertz detector based on the graphene nanomesh was designed and the terahertz detector was developed. The electrical characteristics of the graphene nanomesh were tested, which verified the regulating effect of the graphene nanomesh on the electronic energy gap. A 
test system was built to test the developed detector, suggesting that the $\mathrm{THz}$ detection sensitivity of the detector can reach $2.5 \mathrm{~A} / \mathrm{W}$ at room temperature.

Author Contributions: Y.W., G.C. and Z.W. conceived and designed the study; Y.X. and G.C. performed the theory; Y.Z., W.Y. and G.L. performed the experiments; W.Y., Y.X. and J.S. analyzed the experimental data; Y.Z., W.Y. and Y.W. wrote the paper; Y.W. and Z.W. provided guidance and modification of the paper. All authors have read and agreed to the published version of the manuscript.

Funding: This research work was financially supported by the Chongqing Research Program of Basic Research and Frontier Technology (NO: cstc2018jcyjAX0519).

Conflicts of Interest: The authors declare no conflict of interest.

\section{References}

1. Wang, R.; Ren, X.; Yan, Z.; Jiang, L.; Sha, W.; Shan, G. Graphene based functional devices: A short review. Front. Phys. 2019, 14, 13603. [CrossRef]

2. Jacob, A.; Xie, R.; Sung, M.; Liebmann, L.; Lee, R.; Taylor, B. Scaling Challenges for Advanced CMOS Devices. Int. J. High Speed Electron. Syst. 2017, 26, 1740001. [CrossRef]

3. Buron, J.; Pizzocchero, F.; Jepsen, P.; Ptersen, D.; Caridad, J.; Jessen, B.; Booth, T.; Begglid, P. Graphene mobility mapping. Sci. Rep. 2015, 5, 12305. [CrossRef] [PubMed]

4. $\quad$ Banszerus, L.; Schmitz, M.; Engels, S.; Dauber, J.; Oellers, M.; Haupt, F.; Watanabe, K.; Taniguchi, T.; Beschoten, B.; Stampfer, C. Ultrahigh-mobility graphene devices from chemical vapor deposition on reusable copper. Sci. Adv. 2015, 1, e1500222. [CrossRef] [PubMed]

5. Ren, W.; Cheng, H. The global growth of graphene. Nat. Nanotechnol. 2014, 9, 726-730. [CrossRef] [PubMed]

6. Bandurin, D.; Gayduchencko, I.; Cao, Y.; Moskotin, M.; Principi, A.; Grigorieva, I.; Goltsman, G.; Fedorov, G.; Svintsov, D. Dual Origin of Room Temperature Sub-Terahertz Photoresponse in Graphene Field Effect Transistors. Appl. Phys. Lett. 2018, 112, 141101. [CrossRef]

7. Vicarelli, L.; Vitiello, M.; Coquillat, D.; Lombardo, A.; Derrari, A.; Knap, W.; Polini, M.; Pellegrini, V.; Tredicucci, A. Graphene field-effect transistors as room-temperature terahertz detectors. Nat. Mater. 2012, 11, 865-871. [CrossRef] [PubMed]

8. Feng, W.; Zhang, R.; Cao, J. Progress of terahertz devices based on graphene. Acta Phys. Sin. 2015, 64, 229501.

9. Docherty, C.; Johnston, M. Terahertz Properties of Graphene. J. Infrared Millim. Terahertz Waves 2012, 33, 797-815. [CrossRef]

10. Hesler, J.; Prasankumar, R.; Tignon, J. Advances in terahertz solid-state physics and devices. J. Appl. Phys. 2019, 126, 110401. [CrossRef]

11. Spirito, D.; Coquillat, D.; Bonis, S.; Lombardo, A.; Bruna, M.; Ferrari, A.; Pellgegrini, V.; Tredicucci, A.; Knap, W.; Viteillo, M. High performance bilayer-graphene Terahertz detectors. Appl. Phys. Lett. 2014, 104, 97-105. [CrossRef]

12. Otsuji, T.; Tombet, S.; Satou, A.; Fukidome, H.; Suemitsu, M.; Sano, E.; Popov, V.; Ryzhii, M.; Ryzhii, V. Graphene materials and devices in terahertz science and technology. MRS Bull. 2012, 37, 1235-1243. [CrossRef]

13. Qin, H.; Sun, J.; Liang, S.; Li, X.; Yang, X.; He, Z.; Yu, C.; Feng, Z. Room-temperature, low-impedance and high-sensitivity terahertz direct detector based on bilayer graphene field-effect transistor. Carbon 2017, 116, 760-765. [CrossRef]

14. Yuan, W.; Wen, Z.; Li, M.; Chen, L.; Chen, G.; Ruan, D.; Gao, Y. Energy gap of novel edge-defected graphene nanoribbons. Jpn. J. Appl. Phys. 2016, 55, 085101. [CrossRef]

15. Li, X.; Cai, W.; An, J.; Kim, S.; Nah, J.; Yang, D.; Piner, R.; Velamakanni, A.; Jung, I.; Tutuc, E.; et al. Large-area synthesis of high-quality and uniform graphene films on copper foils. Science 2009, 324, 1312. [CrossRef] [PubMed]

16. Li, X.; Cai, W.; Colombo, L.; Ruoff, R. Evolution of graphene growth on Cu and Ni studied by carbon isotope labeling. Nano Lett. 2009, 9, 4268-4272. [CrossRef] [PubMed]

17. Yuan, W.; Li, M.; Wen, Z.; Sun, Y.; Ruan, D.; Zhang, Z.; Chen, G.; Gao, Y. The Fabrication of Large-Area, Uniform Graphene Nanomeshes for High-Speed, Room-Temperature Direct Terahertz Detection. Nanoscale Res. Lett. 2018, 13, 190. [CrossRef] [PubMed]

18. Zhu, W.; Neumayer, D.; Perebeinos, V.; Avouris, P. Silicon nitride gate dielectrics and bandgap engineering in graphene layers. Nano Lett. 2010, 10, 3572-3576. [CrossRef] [PubMed] 